\title{
IN SILICO SCREENING OF ANTIMALARIAL FROM INDONESIAN MEDICINAL PLANTS DATABASE TO PLASMEPSIN TARGET
}

\author{
EKO ADITYA RIFAI, HAYUN HAYUN, ARRY YANUAR*
}

Department of Pharmaceutical Chemistry, Faculty of Pharmacy, Universitas Indonesia, Depok, Indonesia. Email: arry.yanuar@ui.ac.id

Received: 21 April 2017, Revised and Accepted: 13 July 2017

\section{ABSTRACT}

Objective: Malaria is a disease that impacts millions of people annually. Among the enzymes, plasmepsin is the main enzyme in the plasmodium life cycle that degrades hemoglobin during the erythrocytic phase in the food vacuole. Recently, pharmaceutical industries have been trying to develop therapeutic agents that can cure malaria through the discovery of new plasmepsin inhibitor compounds. One of the developing approaches is the in silico method.

Methods: The chosen in silico screening method in this experiment is a structure-based screening using GOLD software and the Indonesian medicinal plants database.

Results: From ten in silico screening runs, three of the compounds always ranked in the top ten. These three compounds are trimyristin, cyanidin 3,5-di-(6-malonylglucoside), and isoscutellarein 4'-methyl ether 8-(6"-n-butylglucuronide). Another compound that emerged with high frequency is cyanidin 3,5-di-(6-malonylglucoside).

Conclusions: Based on the results obtained from this screening, 11 inhibitor candidates are expected to be developed as antimalarial. These compounds are trimyristin; cyanidin 3,5-di-(6-malonylglucoside); isoscutellarein 4'-methyl ether 8-(6"-n-butylglucuronide); cyanidin 3-(6"-malonylglucoside)-5glucoside; multifloroside; delphinidin 3-(2-rhamnosyl-6-malonylglucoside); delphinidin 3-(6-malonylglucoside)-3',5'-di-(6-p-coumaroylglucoside); cyanidin 3-[6-(6-sinapylglucosyl)-2-xylosylgalactoside; kaempferol 3-glucosyl-(1-3)-rhamnosyl-(1-6)-galactoside; sanggenofuran A; and lycopene with a GOLD score range from 78.4647 to 98.2836. Two of them, Asp34 and Asp214, bind with all residues in the catalytic site of plasmepsin.

Keywords: Antimalarial, In silico screening, Indonesian medicinal plants database, Plasmepsin.

(C) 2017 The Authors. Published by Innovare Academic Sciences PvtLtd.This is an open access article under the CC BY license (http://creativecommons. org/licenses/by/4. 0/) DOI: http://dx.doi.org/10.22159/ajpcr.2017.v10s5.23115

\section{INTRODUCTION}

Every year, malaria, a disease caused by plasmodium, affects billions of people; over 2.5 million lose their lives. There are four species of plasmodium, namely, Plasmodium falciparum, Plasmodium ovale, Plasmodium vivax, and Plasmodium malariae. Of those four, P. falciparum accounts for over $95 \%$ of all malaria cases, mainly in Africa, and children under 5 years of age are among the most vulnerable to malaria infection [1]. It has been shown that hemoglobin catabolism, which takes place in an acidic food vacuole, is essential for parasite survival, both in culture and in animal models [2-4]. Besides asparticproteases, three cysteine proteases (falcipain-1, -2 , and -3 ) and one metalloprotease (falcilysin) have also been identified to digest hemoglobin in the food vacuole [5-8]. To break hemoglobin down, several enzymes are involved, but plasmepsins are known to be vital for this function, as they initiate the catalytic breakdown of hemoglobin [9]. Two aspartic proteases of $P$. falciparum have been implicated in the initial steps of the hemoglobin degradation process. The first protease, plasmepsin I (Plm I), appears to make an initial strategic cleavage that presumably leads to an unraveling of the native hemoglobin structure such that further proteolysis can rapidly proceed. Plasmepsin II (Plm II), the second aspartic protease, is capable of cleaving native hemoglobin, but is more active against denatured or fragmented globin, such as that produced by the action of Plm I [10-12]. Plm I and Plm II make the first strategic cleavage of hemoglobin between Phe33 and Leu34 of the R-chain, resulting in protein unfolding and release of the heme moiety. Subsequent degradation steps are catalyzed by the cysteine protease falcipain, the metalloprotease falcilysin, and cytoplasmic aminopeptidases [13-16]. These complexes reveal key conserved hydrogen bonds between the inhibitor and the binding-cavity residues, notably with the flap residues Val78 and Ser79, the catalytic dyad Asp34 and Asp214, and the residues Ser218 and Gly36 that are in proximity to the catalytic dyad [17]. The catalytic mechanism of plasmepsin II is that the Asp34 and Asp214 residues coordinate a water molecule that following abstraction of a proton by Asp214, attacks the Phe33-Leu34 peptide bond of the $\alpha$-chain in host hemoglobin [18].

As a mega-biodiversity nation, Indonesia ranks second in the world after Brazil. If marine biota is included, Indonesia moves to the first position. About 40,000 species of plants live on Earth, of which 30,000 species live in the Indonesian archipelago. Among the 30,000 plant species in the Indonesian archipelago, at least 9,600 species of plants are known to have pharmacological activity [19]. Previous research showed that the medicinal plants database and three-dimensional structure of the active compounds from medicinal plants in Indonesia have been prepared [20]. This database is supposed to be valuable compounds as an inhibitor for various diseases and targets, including plasmepsin in malaria.

In silico screening of small-molecule compounds against protein targets implicated in a disease of interest has been widely used to discover potential inhibitors. The efficacy and efficiency of multi-target in silico screening provides the potential to considerably reduce time, effort, and cost to obtain promising candidates for drug development [21]. In silico screening orients and scores a library of small molecules in the binding site of a protein, ranking the compounds from best to worst. Often, only a few of the top-scoring hits selected for experimental testing actually bind [22]. Regarding these characteristics, in silico screening will be favorably conducted to search potential plasmepsin inhibitors from the Indonesian medicinal plants database. This research aimed to gain candidates of inhibitor compounds in silico screening using GOLD [23] 
software and ligands of the Indonesian medicinal plants database that originated from natural products which have an activity of inhibiting plasmepsin as the initial step of the antimalarial discovery process. 11 potential compounds from the database will be ranked and considered as hits.

\section{METHODS}

This experiment was conducted using literature study and in silico screening by molecular docking (structure-based in silico screening). The applied experimental design was as follows.

\section{Plasmepsin structure preparation}

Selecting a suitable plasmepsin structure for the binding target was the initial step of this research. Plasmepsin II (1LEE) was chosen. This plasmepsin structure was obtained from the Protein Data Bank [24] website as *.pdb file format. Plasmepsin II or 1LEE has one subunit (monomer), which is subunit $\mathrm{A}$, and one ligand, which is 4 -amino- $\mathrm{N}$ \{4-[2-(2,6-dimethyl-phenoxy)-acetylamino]-3-hydroxy-1-isobutyl5-phenyl-pentyl\} benzamide (R36), that is, gained through $\mathrm{x}$-ray diffraction [17]. The structure was separated from solvent and ligand or non-standard residue using UCSF Chimera [25] software, then was optimized using Vega ZZ [26], including separation of the water molecule and the addition of a hydrogen atom. Afterward, Gasteiger partial charges were added, and AutoDock force field was applied. The last step was minimization using the steepest descent and conjugate gradient 100 and 1,000 steps, respectively.

\section{Ligand file format preparation}

The Indonesian medicinal plants compounds structure were obtained from the HerbalDB [20]. Research was conducted beforehand to

Table 1: Docking results of positive control compounds using GOLD with slow speed and plasmepsin as target

\begin{tabular}{lllll}
\hline $\begin{array}{l}\text { Rank based on } \\
\text { GOLD score }\end{array}$ & Name & $\begin{array}{l}\text { GOLD score } \\
\text { average }(\mathbf{n = 5 )}\end{array}$ & SD & CV (\%) \\
\hline 1 & Pepstatin* & 86.89 & 1.89 & 2.17 \\
2 & R37** & 73.10 & 4.55 & 6.22 \\
3 & R36** & 71.04 & 3.42 & 4.82 \\
4 & TIT** $^{* *}$ & 68.24 & 11.32 & 16.59 \\
5 & 5FE** & 66.20 & 8.61 & 13.00 \\
6 & Halofantrin* & 65.82 & 2.06 & 3.13 \\
7 & 5FP** & 63.73 & 3.73 & 5.86 \\
8 & IH4** & 63.25 & 3.06 & 4.83 \\
9 & EH5** & 62.40 & 11.55 & 18.52 \\
10 & IVS** & 62.20 & 4.75 & 7.64 \\
11 & Norstatin* & 61.32 & 1.41 & 2.30 \\
\hline *ositive controls downloaded from PubChem Compound, & ** positive controls
\end{tabular}

extracted from Protein Data Bank generate the database 3D structure using Vega ZZ script "2D to 3D." This structure was then optimized by adding hydrogen with the "generic organic" and "after each heavy atom" option. After adding hydrogen to the structure, minimization was done using the steepest descent and conjugate gradient, 1,000 steps for each method. The last step was adding Gasteiger partial charges and applying AutoDock force field with AutoDock Tools [27].

\section{Molecular docking protocol validation}

As the center of molecular docking target, the ligand binding site coordinates were defined using GOLD. The coordinates of the binding site were $\mathrm{x}=31.7977 ; \mathrm{y}=33.2087$; and $\mathrm{z}=12.3365$ in the plasmepsin target. After being defined, these coordinates were used for validating the protocol using the 11 positive control compounds stated in Table 1 by conducting preliminary docking. The positive controls used are inhibitors of plasmepsin gained from the PubChem Compound [28] website and separated ligands from the Protein Data Bank website. To conduct molecular docking, the data format of the downloaded molecule from PubChem Compound must be converted first from a 2D structure to 3D, and it eventually changes the extension type from *.sdf into *.mol. To do this conversion, Vega ZZ was employed. This preliminary docking was conducted with three different speeds (slow, medium, and fast), five times for each speed, using GOLD.

\section{Indonesian medicinal plants database in silico screening}

In silico screening was conducted 10 times using the GOLD wizard model with the best parameters of the positive control compounds orientation docking. Docking parameter was set at $15 \AA$ radius, $10 \mathrm{GA}$ runs, 10 numbers of solutions, GOLD Score scoring function, and the slow GA search option.

\section{Analysis and visualization of protein-ligand interaction}

The 11 best compounds screened were visualized using GOLD and PyMOL [29] software. The GOLD docking result was saved in *.conf and *.mol formats that can be opened using GOLD and PyMOL. In GOLD, the best conformation of these top 11 compounds was observed, as well as the hydrogen bond between ligand and protein. PyMOL was then employed to process the molecular docking result data to be visualized later.

\section{RESULTS AND DISCUSSION}

Validation of molecular docking protocol using preliminary docking was conducted five times $(\mathrm{n}=5)$ for each speed (slow, medium, and fast) with three positive control compounds from the PubChem A compound known as a plasmepsin inhibitor, and eight positive control compounds extracted from the Protein Data Bank. The results were ranked based on GOLD Score, as shown in Table 1. After obtaining the best parameter from preliminary docking, in silico screening was conducted using the Indonesian medicinal plants database (http://herbaldb.farmasi.

Table 2: Top 11 compounds docked by GOLD based on rank as top ten from each run

\begin{tabular}{|c|c|c|c|c|c|c|}
\hline Rank & Name & $\mathbf{n}$ & $\begin{array}{l}\text { GOLD } \\
\text { score } \\
\text { average }\end{array}$ & SD & CV (\%) & Plant(s) source [20] \\
\hline 1 & Trimyristin & 9 & 85.4396 & 4.9025 & 5.7379 & Aleurites moluccana, Myristica fragrans \\
\hline 2 & Cyanidin 3,5-di-(6-malonylglucoside) & 9 & 84.4627 & 3.6624 & 4.3362 & Thymus serpyllum \\
\hline 3 & Isoscutellarein 4'-methyl ether 8-(6"-n-butylglucuronide) & 9 & 80.8250 & 1.6951 & 2.0972 & Helicteres isora \\
\hline 4 & Cyanidin 3-(6"-malonylglucoside)-5-glucoside & 7 & 83.1239 & 2.2321 & 2.6852 & Thymus serpyllum \\
\hline 5 & Multifloroside & 7 & 82.3070 & 2.5021 & 3.0399 & Jasminum multiflorum \\
\hline 6 & Delphinidin 3-(2-rhamnosyl-6-malonylglucoside) & 6 & 87.8589 & 3.1734 & 3.6119 & Clitoria ternatea \\
\hline 7 & Delphinidin & 4 & 92.5770 & 6.0155 & 6.4979 & Clitoria ternatea \\
\hline 8 & 3-(6-malonylglucoside)-3',5'-di-(6-p-coumaroylglucoside) & 4 & 845243 & 40815 & 48288 & Anium aravenlons Foeniculum vulaaro \\
\hline 9 & Kaempferol 3-glucosyl-(1-3)-rhamnosyl-(1-6)-galactoside & 4 & 82.1026 & 0.9071 & 1.1048 & Camellia sinensis \\
\hline 10 & Sanggenofuran A & 4 & 81.1762 & 1.8186 & 2.2404 & Morus australis \\
\hline 11 & Lycopene & 4 & 81.0619 & 1.6951 & 2.0911 & $\begin{array}{l}\text { Brassica napus, Diospyros kaki, } \\
\text { Momordica charantia, Psidium guajava }\end{array}$ \\
\hline
\end{tabular}


ui.ac.id) (Herbal db) [20]. The top 11 compounds are provided in Table 2. The average GOLD scores range from 80.8250 to 92.5770 .

From ten in silico screening runs, three of the compounds always ranked in the top ten. These three compounds are trimyristin, cyanidin 3,5-di-(6-malonylglucoside), and isoscutellarein 4'-methyl ether 8-(6"-n-butylglucuronide). Trimyristin is a triglyceride from the Euphorbiaceae family, notably from Aleurities moluccana. Trimyristin is also found in Myristica fragrans, which belongs to the Myristicaceae family. Trimyristin is used as a nanoparticle lipid, which is combined with the curcuminoidin malaria treatment [30]. Triglycerides also have roles in malaria treatment as oil-phase transporters of antimalarial, for example, Self-microemulsifying Drug Delivery Systems that alter into a microemulsion after passing through the oral route [31].

Another compound that emerged with the highest frequency is cyanidin 3,5-di-(6-malonylglucoside). This is a flavonoid of the anthocyanin class which belongs to Lamiaceae family, notably from Thymus serpyllum. Anthocyanin is known to have antimalarial properties. It is also contained in Corchorus olitorius and is known to inhibit the malaria parasite $P$. falciparum more than $96 \%$ [32].

The other compound with the same frequency as the above compounds is isoscutellarein 4'-methyl ether 8-(6"-n-butylglucuronide). This is a glucuronide flavonoid from Sterculiaceae family, notably Helicteres isora. This species is known to have cucurbitacin, flavonoids, neolignan, and a derivate of rosmarinic acid. An earlier experience [33] extracted some flavonoids from $H$. isora, which are isoscutellarein 4'-methyl ether 8-0- $\beta$ D-glucuronide; isoscutellarein 4'-methyl ether 8-0- $\beta$-D-glucuronide 6 "-n-butyl ester; isoscutellarein 4'-methyl ether 8-0- $\beta$-D-glucuronide 2 "-sulfate; isoscutellarein 4'-methyl ether 8-0- $\beta$-D-glucuronide 2 ", 4 "-disulfate; and Isoscutellarein 8-0- $\beta$-D-glucuronide 2 ", 4"-disulfate.

Most of the top 11 compounds belong to the flavonoid group or its glycol side. Natural and synthetic flavonoids show antimalarial activity [34]. The other experiment [35] also showed that flavonoids from Artemisia annua have in vitro antiplasmodial property, with $\mathrm{IC}_{50}$ score $2.3-6.5 \times 10^{-5} \mathrm{M}$. Flavonoids can also be combined with artemisinin to treat malaria by increasing artemisinin activity [36]. This can be a consideration in the development of antimalarial from flavonoidderived compounds due to their potential to inhibit plasmepsin. It is also valuable to conduct in vitro research regarding this potency to find the exact correlation between these compounds with their properties as antimalarial, especially as plasmepsin inhibitor.

The best 11 candidates of plasmepsin inhibitors were visualized and analyzed using GOLD and PyMOL. This showed that delphinidin 3-(2-rhamnosyl-6-malonylglucoside) and isoscutellarein 4'-methyl ether 8-(6"-n-butylglucuronide) bind with all catalytic residues in plasmepsin, which are Asp34 and Asp214. Delphinidin 3-(2-rhamnosyl6-malonylglucoside) also binds with Gly36, Tyr192, Ser215, and Ser218 residues. This compound has a hydrogen bond with three residues in the plasmepsin active site, which are Asp34, Gly36, and Asp214. Gly36 residue is an active site that is in proximity to the catalytic dyad, which is Asp34 and Asp214. In the hemoglobin degradation process, Asp34 and Asp214 residues coordinate a water molecule that following abstraction of a proton by Asp214, attacks the Phe33-Leu34 peptide bond of the $\alpha$-chain in host hemoglobin [18]. This bond will inhibit the substrate of plasmepsin to bind with the active site of the enzyme. Due to the inhibition of two catalytic dyads of plasmepsin by delphinidin 3-(2-rhamnosyl-6-malonylglucoside), the hemoglobin degradation process will also be inhibited, and so will malaria emergence. The pose of this compound is shown in Fig. 1.

Isoscutellarein 4'-methyl ether 8-(6"'-n-butylglucuronide) binds with plasmepsin in Asp34, Ser79, Asp214, Gly216, and Thr217. Like delphinidin 3-(2-rhamnosyl-6-malonylglucoside), which has a binding site in the plasmepsin catalytic dyad, isoscutellarein 4'-methyl ether 8-(6"-n-butylglucuronide) also binds with two residues in catalytic

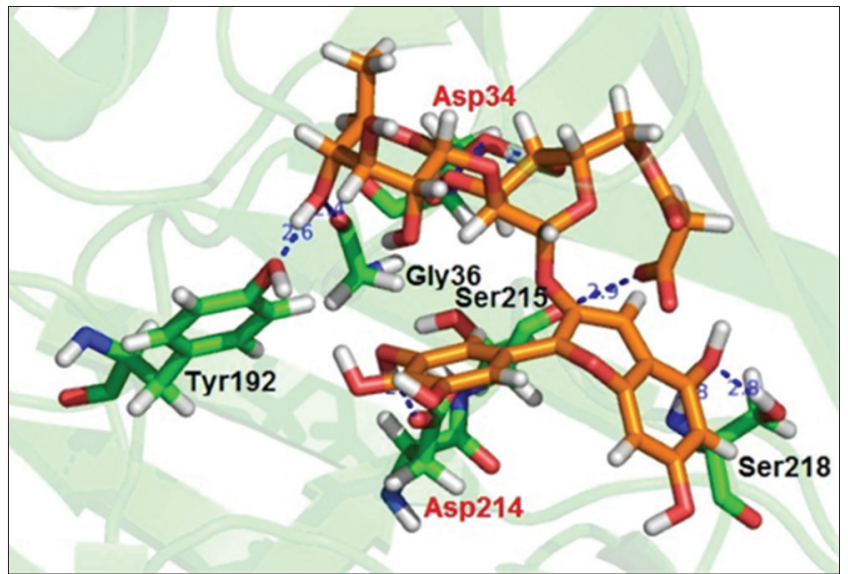

Fig. 1: Interaction of delphinidin 3-(2-rhamnosyl-6malonylglucoside) (orange) with some amino acid residues (green) in plasmepsin. The red font shows the catalytic dyad Asp34 and Asp214

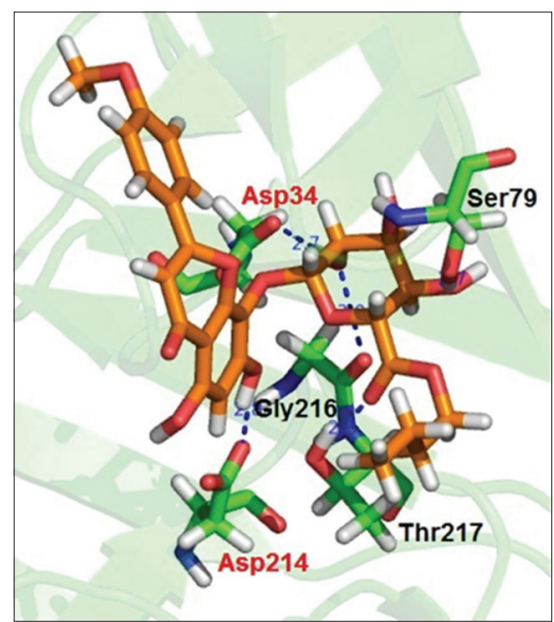

Fig. 2: Interaction of isoscutellarein 4'-methylether 8-(6"-n-butylglucuronide) (orange) with some amino acid residues (green) in plasmepsin. The red font shows the catalytic dyad Asp34 and Asp214

dyads, Asp34, and Asp214, so it can inhibit substrate binding with the enzyme. This binding disarms the enzyme so it cannot catalyze a reaction in the host hemoglobin degradation process. The pose of this compound shown in Fig. 2.

\section{CONCLUSION}

The 11 potential inhibitors against plasmepsin obtained from the in silico screening of the Indonesian medicinal plants database, such as trimyristin; cyanidin 3,5-di-(6-malonylglucoside); isoscutellarein 4'-methyl ether 8-(6"-n-butylglucuronide); cyanidin 3-(6"-malonylglucoside)-5-glucoside; multifloroside; delphinidin 3-(2-rhamnosyl-6-malonylglucoside); delphinidin 3-(6-malonylglucoside)-3',5'-di-(6-p-coumaroylglucoside); cyanidin 3-[6-(6-sinapylglucosyl)-2-xylosylgalactoside; kaempferol 3-glucosyl(1-3)-rhamnosyl-(1-6)-galactoside; sanggenofuran A; and lycopene, have the prospective possibility for further investigation as leading antimalarial compounds.

\section{REFERENCES}

1. Wongsrichanalai C, Pickard AL, Wernsdorfer WH, Meshnick SR. Epidemiology of drug-resistant malaria. Lancet Infect Dis 2002;2(4):209-18. 
2. Rosenthal PJ. Plasmodium falciparum: Effects of proteinase inhibitors on globin hydrolysis by cultured malaria parasites. Exp Parasitol 1995;80(2):272-81.

3. Olson JE, Lee GK, Semenov A, Rosenthal PJ. Antimalarial effects in mice of orally administered peptidyl cysteine protease inhibitors. Bioorg Med Chem 1999; 7:633-8.

4. Boss C, Richard-Bildstein S, Weller T, Fischli W, Meyer S, Binkert C. Inhibitors of the Plasmodium falciparum parasite aspartic protease plasmepsin II as potential antimalarial agents. Curr Med Chem 2003;10(11):883-907.

5. Salas F, Fichmann J, Lee GK, Scott MD, Rosenthal PJ. Functional expression of falcipain, a Plasmodium falciparum cysteine proteinase, supports its role as a malarial hemoglobinase. Infect Immun 1995;63(6):2120-5.

6. Shenai BR, Sijwali PS, Singh A, Rosenthal PJ. Characterization of native and recombinant falcipain-2, a principal trophozoite cysteine protease and essential hemoglobinase of Plasmodium falciparum. J Biol Chem 2000;275(37):29000-10.

7. Sijwali PS, Shenai BR, Gut J, Singh A, Rosenthal PJ. Expression and characterization of the Plasmodium falciparum haemoglobinase falcipain-3. Biochem J 2001;360:481-9.

8. Eggleson KK, Duffin KL, Goldberg DE. Identification and characterization of falcilysin, a metallopeptidase involved in hemoglobin catabolism within the malaria parasite Plasmodium falciparum. J Biol Chem 1999;274(45):32411-7.

9. Bhaumik P, Xiao H, Parr CL, Kiso Y, Gustchina A, Yada RY, et al. Crystal structures of the histo-aspartic protease (HAP) from Plasmodium falciparum. J Mol Biol 2009;388(3):520-40.

10. Francis SE, Gluzman IY, Oksman A, Knickerbocker A, Mueller R, Bryant ML, et al. Molecular characterization and inhibition of a Plasmodium falciparum aspartic hemoglobinase. EMBO J 1994;13(2):306-17.

11. Gluzman IY, Francis SE, Oksman A, Smith CE, Duffin KL, Goldberg DE. Order and specificity of the Plasmodium falciparum hemoglobin degradation pathway. J Clin Invest 1994;93(4):1602-8.

12. Dame JB, Reddy GR, Yowell CA, Dunn BM, Kay J, Berry C. Sequence, expression and modeled structure of an aspartic proteinase from the human malaria parasite Plasmodium falciparum. Mol Biochem Parasitol 1994:64(2):177-90.

13. Banerjee R, Liu J, Beatty W, Pelosof L, Klemba M, Goldberg DE. Four plasmepsins are active in the Plasmodium falciparum food vacuole, including a protease with an active-site histidine. Proc Natl Acad Sci U S A 2002;99(2):990-5

14. Goldberg DE. Hemoglobin Degradation. Curr Top Microbiol Immunol 2005;295:275-91.

15. Goldberg DE, Slater AF, Beavis R, Chait B, Cerami A, Henderson GB. Hemoglobin degradation in the human malaria pathogen Plasmodium falciparum: A catabolic pathway initiated by a specific aspartic protease. J Exp Med 1991;173(4):961-9.

16. Klemba M, Goldberg DE. Biological roles of proteases in parasitic protozoa. Annu Rev Biochem 2002;71:275-305

17. Asojo OA, Afonina E, Gulnik SV, Yu B, Erickson JW, Randad R, et al. Structures of Ser205 mutant plasmepsin II from Plasmodium falciparum at $1.8 \mathrm{~A}$ in complex with the inhibitors rs367 and rs370. Acta Crystallogr D Biol Crystallogr 2002;58:2001-8.

18. Gupta D, Yedidi RS, Varghese S, Kovari LC, Woster PM. Mechanism- based inhibitors of the aspartyl protease plasm II as potential antimalarial agents. J Med Chem 2010;53(10):4234-47.

19. Departemen Kesehatan Republik Indonesia. Kebijakan Obat Tradisional Nasional Tahun 2007: Keputusan Menteri Kesehatan RI No. 381/MENKES/SK/III/2007. Jakarta: Ditjen Bina Kefarmasian dan Alat Kesehatan Departemen Kesehatan RI; 2007. Available from: http://www.binfar.depkes.go.id/dat/lama/1206328790_Buku\%20 Kebijakan\%20Obat\%20Tradisional\%20Nasional\%20Tahun $\% 20$ 2007.pdf.

20. Yanuar A, Mun'im A, Lagho AB, Syahdi RR, Rahmat M, Suhartanto H. Medicinal plants database and three dimensional structure of the chemical compounds from medicinal plants in Indonesia. Int J Comput Sci $2011 ; 8(5): 180-3$.

21. Jenwitheesuk E, Horst JA, Rivas KL, Van Voorhis WC, Samudrala R. Novel paradigms for drug discovery: Computational multitarget screening. Trends Pharmacol Sci 2008;29(2):62-71

22. Irwin JJ. Community benchmarks for virtual screening. J Comput Aided Mol Des 2008;22(3-4):193-9.

23. Hartshorn MJ, Verdonk ML, Chessari G, Brewerton SC, Mooij WT, Mortenson PN, et al. Diverse, high-quality test set for the validation of protein-ligand docking performance. J Med Chem 2007;50(4):726-41.

24. Berman HM, Westbrook J, Feng Z, Gilliland G, Bhat TN, Weissig H, et al. The protein data bank. Nucleic Acids Res 2000;28(1):235-42.

25. Pettersen EF, Goddard TD, Huang CC, Couch GS, Greenblatt DM, Meng EC, et al. UCSF chimera-a visualization system for exploratory research and analysis. J Comput Chem 2004;25(13):1605-12.

26. Pedretti A, Mazzolari A, Vistoli G, Vega ZZ. A versatile toolkit for drug design and protein modelling. J Comput Aided Mol Des 2004;18:167-73.

27. Syahdi RR, Mun'im A, Suhartanto H, Yanuar A. Virtual screening of Indonesian herbal database as HIV-1 reverse transcriptase inhibitor. Bioinformation 2012;8(24):1206-10

28. Bolton EE, Wang Y, Thiessen PA, Bryant SH. PubChem: Integrated platform of small molecules and biological activities. Annu Rep Comput Chem 2008;4:217-41

29. DeLano WL. The PyMOL Molecular Graphics System, Version 0.99. San Carlos, CA: DeLano Scientific; 2002.

30. Nayak AP, Tiyaboonchai W, Patankar S, Madhusudhan B, Souto EB. Curcuminoids-loaded lipid nanoparticles: Novel approach towards malaria treatment. Colloids Surf B Biointerfaces 2010;81(1):263-73.

31. Santos-Magalhães NS, Mosqueira VC. Nanotechnology applied to the treatment of malaria. Adv Drug Deliv Rev 2010;62(4-5):560-75.

32. Morris JB, Wang ML. Anthocyanin and potential therapeutic traits in Clitoria, Desmodium, Corchorus, Catharanthus, and Hibiscus Species. Acta Hortic 2007;756:381-8.

33. Kamiya K, Saiki Y, Hama T, Fujimoto Y, Endang H, Umar M, et al. Flavonoid glucuronides from Helicteres isora. Phytochemistry 2001;57(2):297-301.

34. Lim SS, Kim H, Lee D. In vitro antimalarial activity of flavonoid and chalcones. Bull Korean Chem Soc 2007;28:2495-7.

35. Liu KC, Yang SL, Roberts MF, Elford BC, Phillipson JD. Antimalarial activity of Artemisia annua flavonoids from whole plants and cell cultures. Plant Cell Rep 1992;11(12):637-40.

36. Ferreira JF, Luthria DL, Sasaki T, Heyerick A. Flavonoids from Artemisia annua L. as antioxidants and their potential synergism with artemisinin against malaria and cancer. Molecules 2010;15(5):3135-70. 